\title{
Initial assessment of single- and dual-frequency BDS-3 RTK positioning
}

Yunbin Yuan ${ }^{1 *}$, Xiaolong $\mathrm{Mi}^{1,2}$ and Baocheng Zhang ${ }^{1}$

\begin{abstract}
The BeiDou navigation satellite system with global coverage (BDS-3) has been fully operational since July 2020 and provides comprehensive services to global users. BDS-3 transmits several new navigational signals based on the signals inherited from the BeiDou navigation satellite (regional) system (BDS-2). Previous studies focused on the positioning performance of BDS-2 plus BDS-3 and that of combining BDS-3 and other Global Navigation Satellite Systems (GNSSs), but there was no in-depth discussion on the positioning performance of the BDS-3-only. In this contribution, the BDS-3-only Real-Time Kinematic (RTK) positioning is analysed using the data collected in zero and short baselines in Wuhan, China. The RTK model based on Single-Differenced is first presented, and the BDS-3-only RTK positioning in cases of single and dual-frequencies is evaluated with the model in terms of the empirical integer ambiguity resolution success rates and positioning accuracy. Our numerical tests suggest two major findings. First, the positioning performance for the B1I and B3I retained from BDS-2 and the new frequency B1C is comparable, while that for the new frequency B2a is poorer. Second, the positioning performance of the new frequency combination of the B1C+B2a is not as good as that of the B1C only, owing to the unrealistic stochastic model used.
\end{abstract}

Keywords: BDS-3, Global navigation satellite system, Real-time kinematic, Integer ambiguity resolution, Stochastic model

\section{Introduction}

The BeiDou Navigation Satellite System (BDS) is an integrated navigation and communication system developed and operated by China. It provides the navigation services equivalent to Global Positioning System (GPS) while complementing the shortcomings of the traditional means of communication through the original short message communication service (Yang et al. 2018; Wang et al. 2019). BDS was developed in three phases. As a demonstration system, BeiDou navigation demonstration system (BDS-1) already completed its mission and ceased its services (Lv et al. 2020). As a regional system serving the Asia-Pacific region, BDS-2 is currently operating and provides stable Positioning, Navigation,

\footnotetext{
*Correspondence: yybgps@asch.whigg.ac.cn

1 State Key Laboratory of Geodesy and Earth's Dynamics, Innovation Academy for Precision Measurement Science and Technology, Chinese Academy of Sciences, Wuhan, China

Full list of author information is available at the end of the article
}

And Timing (PNT) services with a constellation of five satellites in GEostationary Orbit (GEO), seven in an Inclined GeoSynchronous Orbit (IGSO), and three in Medium-altitude Earth Orbit (MEO) (CSNO 2017; Shi et al. 2020). Furthermore, the entire constellation of BDS-2 broadcasts triple-frequency signals, namely, B1I at $1561.098 \mathrm{MHz}, \mathrm{B} 2 \mathrm{I}$ at $1207.140 \mathrm{MHz}$, and B3I at 1268.520 MHz (CSNO 2018; Odolinski et al. 2014). In the third phase BDS- 3 consists of 30 satellites, including three GEO, 24 MEO and three IGSO satellites, providing reliable PNT services for global users (Yang et al. 2020; CSNO 2019b). BDS-3 retains B1I and B3I of BDS-2 to realise the transition from BDS-2 to BDS-3. On this basis, BDS-3 also transmits three new frequency signals to achieve compatibility and interoperability with other GNSSs, namely, B1C at $1575.42 \mathrm{MHz}, \mathrm{B} 2 \mathrm{a}$ at $1176.45 \mathrm{MHz}$, and B2b at 1207.14 MHz (CSNO 2019a; Gu et al. 2020).

BDS-3 has provided basic services to global users since the end of 2018. Consequently, the study of signal 
quality, precise orbit determination, precise positioning, and timing based on new satellites and new signals has been of great interest among researchers. It has been proven that the code noise of BDS-3 satellites is lower than that of BDS-2 owing to a higher $\mathrm{C} / \mathrm{N}_{0}$ value, while the phase noise level is comparable to that of BDS-2 (Zhang et al. 2019a). The Precise Orbit Determination (POD) for BDS-3 satellites using both L-band satelliteground and Ka-band Inter-Satellite Link (ISL) observations was presented, and it was confirmed that the noise of ISL observations is less than $10 \mathrm{~cm}$ (Xie et al. 2019). The Precise Point Positioning (PPP) using combined BDS-2 and BDS-3 measurements is compared with the PPP using BDS-2 observations only. The results show that the positioning accuracy and convergence time with the combined BDS-2 and BDS-3 are improved to some extent (Jiao et al. 2019; Jin and $\mathrm{Su} 2020$ ). It is preliminarily proved that interoperability of BDS-3 and other GNSSs can be achieved by analysing the Inter-System Biases (ISBs) between their overlapping frequencies (Mi et al. 2019c). A stochastic model of BDS-3 was studied, and it was preliminarily concluded that the signal quality of B1C and B2a was better than that of B1I and B3I (Hou et al. 2019). Analysis of RTK positioning performance based on BDS-3 has preliminarily proven that the positioning accuracy of BDS-3 is higher than that of BDS-2 (Zhang et al. 2019b). However, the number of BDS-3 satellites at that time was relatively small, thus the availability of positioning was not high, and further research on positioning with BDS-3 only has yet to be conducted.

It is worth noting that $\mathrm{He}$ et al. (2014) evaluated the performance of BDS single-epoch RTK positioning, which was based on BDS-2 with two frequencies (B1I and B2I). The primary goal of this study is to evaluate the RTK positioning performance of single- and dualfrequency BDS-3-only positioning. In this study, the RTK model of both single- and dual-frequency positioning based on SD is developed. Then, we divide the frequencies of BDS-3 into two types: the old frequencies retained in BDS-2, including B1I and B3I, and the new frequencies compatible with other GNSSs, including B1C and B2a. The RTK positioning performance of the single- and dual-frequency BDS-3-only positioning for both old and new frequencies will be analysed in terms of positioning accuracy and integer ambiguity resolution success rates.

\section{SD RTK model}

For short baselines of a few kilometers, relative tropospheric and ionospheric delays can be ignored (Mi et al. 2019b; Odolinski et al. 2015b). In this case, the SD RTK model can be expressed as

$$
\begin{aligned}
& p_{12, j}^{s_{*}}(i)=g_{2}^{s_{*}^{T}}(i) \cdot x_{12}(i)+d t_{12}(i)+d_{12, j}^{*}(i)+\varepsilon_{12, j}^{s_{*}} \\
& \phi_{12, j}^{s_{*}}(i)=g_{2}^{s_{*}^{T}}(i) \cdot x_{12}(i)+d t_{12}(i)+\delta_{12, j}^{*}(i)+\lambda_{j} z_{12, j}^{s_{*}}+e_{12, j}^{s_{*}}
\end{aligned}
$$

where $(\cdot)_{12}=(\cdot)_{2}-(\cdot)_{1}$ is the notation for betweenreceiver; $p_{12, j}^{s_{*}}(i)$ and $\phi_{12, j}^{s_{*}}(i)$ denote the vectors of the SD code and phase observations of satellite $s_{*}$ on frequency $j$ respectively; the symbols $x_{12}(i)$ and $g_{2}^{s_{*}^{t}}(i)$ denote the column vector of geometric unknowns and the row vector of the receiver-to-satellite unit vector, respectively; $d t_{12}(i)$, $d_{12, j}^{*}(i)$, and $\delta_{12, j}^{*}(i)$ represent the receiver clock error, code bias, and phase bias, respectively; $\lambda_{j}$ is the wavelength of frequency $j ; z_{12, j}^{s_{*}}$ are SD integer ambiguities; and $\varepsilon_{12, j}^{s_{*}}$ and $e_{12, j}^{s_{*}}$ are the SD random observation noise and unmodeled effects, such as multipath, respectively.

However, Eq. (1) does not have full rank because of the rank deficiency between the columns of the receiver clock and code and phase delays and between the columns of the phase delays and ambiguities (Odolinski et al. 2015a; Mi et al. 2019a). To eliminate the rank deficiency, we must select corresponding benchmarks. After its elimination, the full-rank RTK model can be described as

$$
\begin{aligned}
p_{12, j}^{s_{*}}(i)= & g_{2}^{s_{*}^{T}}(i) \cdot x_{12}(i)+d \tilde{t}_{12}^{*}(i)+\tilde{d}_{12, j}^{*}(i)+\varepsilon_{12, j}^{s_{*}} \\
\phi_{12, j}^{s_{*}}(i)= & g_{2}^{s_{*}^{T}}(i) \cdot x_{12}(i)+d \tilde{t}_{12}^{*}(i)+\delta_{12}^{*}(i) \\
& +\tilde{\delta}_{12, j}^{*}(i)+\lambda_{j} \tilde{z}_{12, j}^{1_{*} s_{*}}+e_{12, j}^{s_{*}}
\end{aligned}
$$

Table 1 Estimable unknown parameters and their interpretations in the short-baseline RTK model

\begin{tabular}{ll}
\hline Notation and interpretation & Estimable parameter \\
\hline$d \tilde{t}_{12}^{*}(i)=d t_{12}^{*}(i)+d_{12,1}^{*}(i)$ & Receiver clock with code delays on $j=1$ \\
$\tilde{d}_{12, j}^{*}(i)=d_{12, j}^{*}(i)-d_{12,1}^{*}(i)$ & Receiver differential code biases (DCBs), where $j \geq 2$ \\
$\delta_{12}^{*}(i)=\delta_{12,1}^{*}(i)-d_{12,1}^{*}(i)+\lambda_{1} z_{12,1}^{1_{*}^{*}}$ & Receiver differential phase and code bias of the first frequency \\
$\tilde{\delta}_{12, j}^{*}(i)=\delta_{12, j}^{*}(i)-\delta_{12,1}^{*}(i)+\lambda_{j} z_{12, j}^{1_{*}}-\lambda_{1} z_{12,1}^{1_{*}^{*}}$ & Receiver differential phase biases (DPBs), where $j \geq 2$ \\
$\tilde{z}_{12, j}^{1_{*}^{*} s_{*}}=z_{12, j}^{s_{*}}-z_{12, j}^{1 *}$ & Double-differenced (DD) integer ambiguities \\
\hline
\end{tabular}


The meanings of the parameters in Eq. (2) are given in Table 1. This model is the basis for evaluating the RTK positioning performance of BDS-3.

\section{Experimental setup}

We deployed four multi-GNSS receivers (two Trimble Alloy receivers and two Septentrio PolaRx5 receivers) in and near the Institute of Geodesy and Geophysics (IGG) in Wuhan, China (114.4 $\mathrm{E}, 33.6^{\circ} \mathrm{N}$ in WGS84). The two Trimble Alloy receivers, IGG01 and IGG03, were connected to a single antenna (South GR3-G3) and placed in the roof-top plant room of building 707 of the IGG. The other two Septentrio PolaRx5s receivers, IGG04 and IGG05, were installed on the roof of a cooperative unit. The data were collected over three consecutive days (June 18-20, 2020) for BDS-3 signals and with a sampling interval of $30 \mathrm{~s}$.

In our BDS-3 RTK analysis, we refer to two independent receiver pairs that form a short baseline and a zero baseline. The relevant characteristics of the experimental datasets are summarised in Table 2. An elevation-dependent weighting function was used, and the standard deviations of the undifferenced phase and code were $3 \mathrm{~mm}$ and $0.3 \mathrm{~m}$, respectively. We used the Detection, Identification and Adaptation (DIA) procedure to eliminate outliers (Teunissen 2018) and the Least-squares AMBiguity Decorrelation (LAMBDA) method to resolve integer ambiguities (Teunissen 1995) in the data analysis. In addition, acceptance tests were employed as part of the ambiguity resolution process. A popular acceptance test, called the ratio test, was used, and the traditional threshold of 2 was used in the test (Teunissen and Verhagen 2009). The number of visible BDS-3 satellites at IGG01 and IGG04 with an elevation cutoff angle of $15^{\circ}$ over $24 \mathrm{~h}$ is shown in Fig. 1. We can see that there are more visible BDS-3 satellites at IGG01 than that at IGG04 because the Septentrio PolaRx5s receivers IGG04 and IGG05 can only observe BDS-3 satellites with PRN less than 36. For the sake of brevity, we only show the RTK results in some of the experimental days, which are representative of all the experimental results.

Table 2 General overview of the characteristics of two experimental receiver pairs

\begin{tabular}{lll}
\hline Receiver pair & $\begin{array}{l}\text { Base } \\
\text { length } \\
\text { (m) }\end{array}$ & Observation period \\
\hline IGG01-IGG03 (Trimble-Trimble) & 0 & 2020, days 170-172 \\
IGG04-IGG05 (Septentrio-Septentrio) & 10 & \\
\hline
\end{tabular}

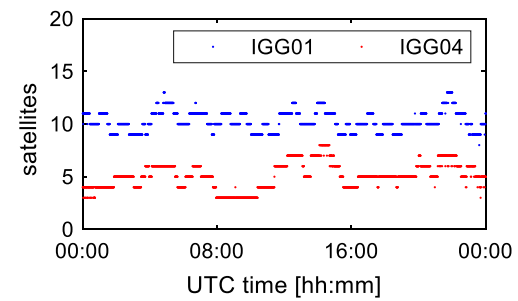

Fig. 1 BDS-3 satellite visibility for IGG01 and IGG04 with an elevation cutoff angle of $15^{\circ}$ on June 20,2020

\section{RTK positioning performance}

In this section, we analyse the RTK positioning performance for four frequencies of BDS-3 and evaluate the performance for dual-frequency combinations. We used a zero baseline and a short one to achieve our goal because the zero baseline can reflect the quality of the satellite signal for the common errors can be eliminated completely, and the short one can reflect the integer ambiguity resolution capability in practice.

\section{Zero-baseline IGG01-IGG03}

The results of ambiguity resolution in terms of the empirical success rates with an elevation cutoff angle of $15^{\circ}$ for BDS-3 single-frequency bands B1C, B2a, B1I, and B3I on day 172 of 2020 are given in Table 3, where the results for dual-frequency combinations, written as the $\mathrm{B} 1 \mathrm{C}+\mathrm{B} 2 \mathrm{a}$ and the B1I+B3I, are also included. The empirical integer ambiguity resolution success rate is defined as the number of epochs with integer ambiguities correctly resolved divided by the total number of epochs. It was computed by comparing the single-epoch estimated integer ambiguities to the reference ambiguities. The reference ambiguities were estimated by a batch solution using BDS-3 with multiple frequencies and by assuming the constant ambiguities over the whole timespan. As we can see from Table 3, except-frequency B2a, the integer ambiguity resolution success rates for the other

Table 3 Empirical integer ambiguity resolution success rates for zero-baseline IGG01-IGG02 based on the BDS-3 data only on day 172 of 2020

\begin{tabular}{ll}
\hline Frequency bands & $\begin{array}{l}\text { Integer ambiguity } \\
\text { resolution success } \\
\text { rate }\end{array}$ \\
\hline B1C & $2880 / 2880=100 \%$ \\
B2a & $2866 / 2880=99.51 \%$ \\
B11 & $2880 / 2880=100 \%$ \\
B31 & $2880 / 2880=100 \%$ \\
B1C+B2a & $2880 / 2880=100 \%$ \\
B11+B31 & $2880 / 2880=100 \%$ \\
\hline
\end{tabular}




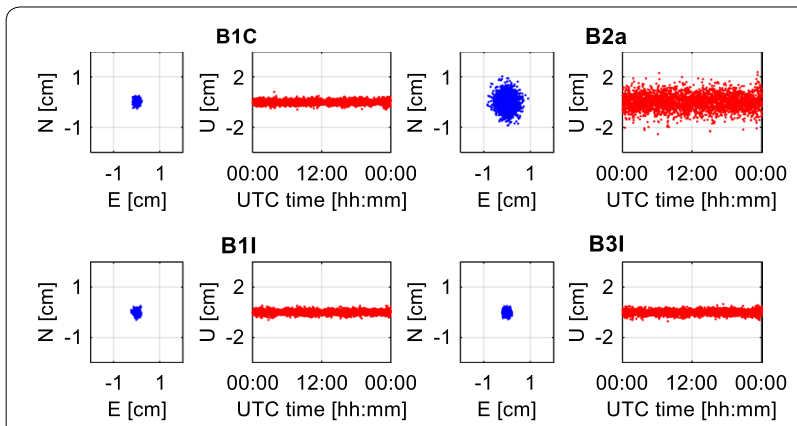

Fig. 2 Horizontal ( $E$ is east direction and $N$ is north direction) position scatters and vertical ( $U$ is up direction) time series of BDS-3 single-frequency B1C, B2a, B1I, and B3I for zero-baseline IGG01-IGG03 with an elevation cutoff angles of $15^{\circ}$ on day 172 of 2020

Table 4 Statistics of the positioning results for zerobaseline IGG01-IGG02

\begin{tabular}{llll}
\hline Frequency bands & \multicolumn{3}{l}{$\begin{array}{l}\text { RMS of the positioning errors } \\
\text { in different directions }(\mathbf{m m})\end{array}$} \\
\cline { 2 - 4 } & $\boldsymbol{E}$ & $\boldsymbol{N}$ & $\boldsymbol{U}$ \\
\hline B1C & 0.6 & 0.6 & 1.5 \\
B2a & 2.3 & 2.6 & 6.1 \\
B11 & 0.6 & 0.6 & 1.4 \\
B31 & 0.7 & 0.7 & 1.7 \\
B1C+B2a & 1.2 & 1.3 & 3.1 \\
B11+B31 & 0.5 & 0.6 & 1.3 \\
\hline
\end{tabular}

three frequencies are all 100\%. Because IGG01-IGG03 is a zero baseline, the errors related to satellites are eliminated. In addition, the errors related to signal propagation, such as tropospheric delay and ionospheric delay, are also eliminated, which makes the resolution of integer ambiguities easy. Therefore, we suspect the reason for the lower success rate for $\mathrm{B} 2 \mathrm{a}$ is due to the relatively poor quality of the signal.

Figure 2 shows the horizontal position scatters and the vertical position time series of baseline IGG01IGG03 based on the BDS-3 on day 172 of 2020 for the four signal cases, i.e., B1C, B2a, B1I, and B3I. Corresponding to the results of integer ambiguity resolution, BIC, B1I, and B3I exhibit a relatively high level of positioning accuracy, while $\mathrm{B} 2 \mathrm{a}$ is much worse. Table 4 provides the information on the positioning accuracy of the fixed single-frequency and single-epoch solutions for the BDS-3 frequencies, as depicted in Fig. 2. The difference in the accuracy of the fixed solution is also obvious, especially in the horizontal components. The root mean square (RMS) of the horizontal components for B2a is at the level of $6 \mathrm{~mm}$, whereas for other frequencies, it is at the level of $1-2 \mathrm{~mm}$. This also supports our suspect

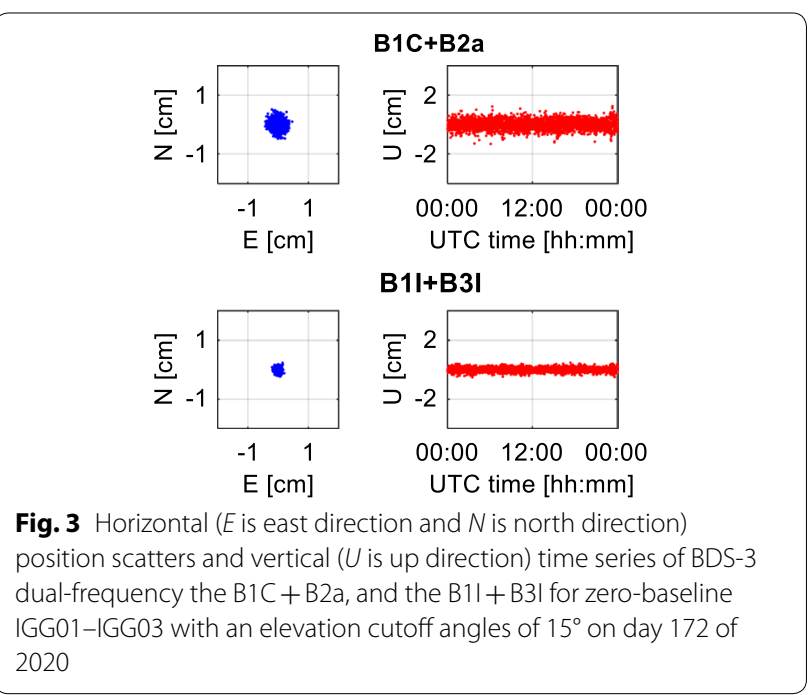

Table 5 Empirical integer ambiguity resolution success rates for short-baseline IGG04-IGG05 based on the BDS-3 data only on day 172 of 2020 (using 2682 epochs with four or more satellites)

\begin{tabular}{ll}
\hline Frequency bands & $\begin{array}{l}\text { Integer ambiguity } \\
\text { resolution success } \\
\text { rate }\end{array}$ \\
\hline B1C & $2536 / 2682=94.6 \%$ \\
B2a & $2646 / 2682=91.3 \%$ \\
B1I & $2875 / 2682=99.8 \%$ \\
B3I & $2878 / 2682=99.9 \%$ \\
B1C+B2a & $2801 / 2682=97.1 \%$ \\
B1I+B31 & $2682 / 2682=100 \%$ \\
\hline
\end{tabular}

mentioned above that the signal quality of B2a is poorer than the others.

Figure 3 shows the horizontal position scatters and vertical position time series for the dual-frequency combinations of the $\mathrm{B} 1 \mathrm{C}+\mathrm{B} 2 \mathrm{a}$ and the $\mathrm{B} 1 \mathrm{I}+\mathrm{B} 3 \mathrm{I}$. The positioning accuracy for the $\mathrm{B} 1 \mathrm{C}+\mathrm{B} 2 \mathrm{a}$ and the $\mathrm{B} 1 \mathrm{I}+\mathrm{B} 3 \mathrm{I}$ is given in Table 4. The RMS values of the north, east, and up components for the $\mathrm{B} 1 \mathrm{C}+\mathrm{B} 2 \mathrm{a}$ are $1.3 \mathrm{~mm}, 1.2 \mathrm{~mm}$, and $3.1 \mathrm{~mm}$, while those for the B1I+B3I are $0.6 \mathrm{~mm}$, $0.5 \mathrm{~mm}$, and $1.3 \mathrm{~mm}$, respectively. Compared with Fig. 2, the positioning accuracy of the frequency combination of the $\mathrm{B} 1 \mathrm{C}+\mathrm{B} 2 \mathrm{a}$ is greatly improved in comparison with that of the single-frequency $\mathrm{B} 2 \mathrm{a}$, but worse than that of the single-frequency B1C. We believe that this is because the same weight is applied to the observations of different frequencies, which leads to the fact that the observations with higher accuracy do not play more important role in the adjustment process. This indicates the importance of establishing a good stochastic model. 


\section{Short-baseline IGG04-IGG05}

Similar to the zero-baseline IGG01-IGG03, the positioning performance of the short-baseline IGG04-IGG05 was also analysed from the aspects of integer ambiguity resolution and positioning accuracy. Table 5 lists the integer ambiguity resolution success rates of different frequencies and combinations for short-baseline IGG04IGG05 on day 172 of 2020 . The results show that the capability of the integer ambiguity resolution for the B1I and B3I is better than that for the B1C and B2a. As we can see, the integer ambiguity resolution success rates for the B1C and B2a are $94.6 \%$ and $91.3 \%$, respectively, while those for B1I and B3I are $99.8 \%$ and $99.9 \%$, respectively. The success rate of the dual-frequency combination is improved compared with that of the single-frequency. The success rate for the $\mathrm{B} 1 \mathrm{C}+\mathrm{B} 2 \mathrm{a}$ is $97.1 \%$, which is $2.6 \%$ and $6.4 \%$ higher than that for $\mathrm{B} 1 \mathrm{C}$ and $\mathrm{B} 2 \mathrm{a}$, respectively.

Figure 4 shows the positioning results of baseline IGG04-IGG05 for BIC, B2a, B1I, and B3I on day 172 of 2020 , including the horizontal position scatters and the vertical position time series. The statistics of the positioning results for short-baseline IGG04-IGG05 is given in Table 6. We confirm and extend our findings in Fig. 3. The positioning accuracy for B1I and B3I is about the same in the three directions, and is slightly higher than that for $\mathrm{B} 1 \mathrm{C}$, while that for $\mathrm{B} 2 \mathrm{a}$ is the worst. The RMS of the horizontal components for B1C, B1I, and B3I are at the level of $10 \mathrm{~mm}$, whereas for $\mathrm{B} 2 \mathrm{a}$, it increased to a level of $20 \mathrm{~mm}$. In addition, the time series of the up component tells that the results for B1I and B3I are more stable than for B1C.

Figure 5 shows the positioning results for the $\mathrm{B} 1 \mathrm{C}+\mathrm{B} 2 \mathrm{a}$ and the $\mathrm{B} 1 \mathrm{I}+\mathrm{B} 3 \mathrm{I}$ on day 172 of 2020 . Compared with the single-frequency results, the positioning accuracy of the dual-frequency combination is improved to some extent. The RMS values of the three components
Table 6 Statistics of the positioning results for shortbaseline IGG04-IGG05

\begin{tabular}{lllr}
\hline Frequency bands & \multicolumn{3}{l}{$\begin{array}{l}\text { RMS of the positioning errors } \\
\text { in different directions }(\mathbf{m m})\end{array}$} \\
\cline { 2 - 4 } & $\boldsymbol{E}$ & $\boldsymbol{N}$ & $\boldsymbol{U}$ \\
\hline B1C & 3.5 & 3.0 & 10.1 \\
B2a & 7.4 & 7.6 & 18.9 \\
B1I & 3.1 & 2.8 & 9.3 \\
B31 & 3.5 & 3.2 & 9.5 \\
B1C+B2a & 2.9 & 3.2 & 8.2 \\
B1I+B31 & 2.1 & 2.1 & 6.6 \\
\hline
\end{tabular}

for the $\mathrm{B} 1 \mathrm{C}+\mathrm{B} 2 \mathrm{a}$ are $3.2 \mathrm{~mm}, 2.9 \mathrm{~mm}$, and $8.2 \mathrm{~mm}$, respectively, while those for $\mathrm{B} 2 \mathrm{a}$ are $7.6 \mathrm{~mm}, 7.4 \mathrm{~mm}$, and $18.9 \mathrm{~mm}$, with the improvement of $57.9 \%, 60.8 \%$, and $56.6 \%$, respectively. The advantages of the dual-frequency combinations are clearly demonstrated in this example. They become more obvious for a longer baseline because of dual-frequency suppression of ionospheric and tropospheric effects, facilitating the resolution of the integer ambiguities.

\section{Conclusion}

BDS-3 has been fully operational since July 2020 and provides comprehensive services to global users. In this study, we first developed an RTK positioning model based on the SD, which is suitable for single, double, and multiple frequencies, and used the S-basis theory to construct its full-rank functional model. On this basis, we applied the model to the RTK positioning of BDS-3 with single and dual frequencies. The main contribution of this work is that for the first time we analysed the RTK

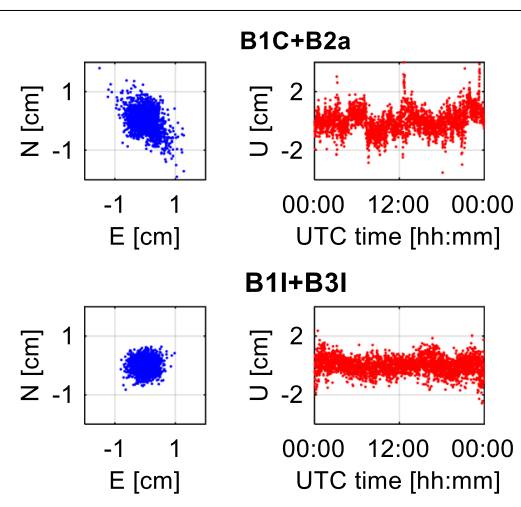

Fig. 5 Horizontal ( $E$ is east direction and $N$ is north direction) position scatters and vertical position ( $U$ is up direction) time series for dual-frequency the $\mathrm{B} 1 \mathrm{C}+\mathrm{B} 2 \mathrm{a}$, and the $\mathrm{B} 1 \mathrm{I}+\mathrm{B} 3 \mathrm{I}$ for short-baseline IGG04-IGG05 with an elevation cutoff angles of $15^{\circ}$ on day 172 of 2020 
performance of BDS-3-only positioning for single- and dual-frequency.

To conduct tests we deployed four receivers in Wuhan, China, capable of receiving BDS-3 signals: two Trimble Alloy receivers and two Septentrio PolaRx5 receivers. We then analysed the RTK positioning performance for single-frequency bands B1C, B2a, B1I, and B3I as well as dual-frequency bands the B1C+B2a and the B1I+B3I using the collected BDS-3 data in terms of integer ambiguity resolution and positioning accuracy. According to the experimental results, we found that the positioning accuracies of single-frequency bands B1C, BII, and B3I were about the same, while that for band B2a was lower. The zero-baseline configuration enables the single-differencing between the receivers to fully eliminate the errors related to satellites and the atmosphere. Therefore, we attributed the poor positioning performance for the single-frequency band B2a to its poor signal quality. In addition, the positioning results for dual-frequency combinations are not always better than those for single-frequency because of the absence of a good stochastic model. For example, the positioning accuracy for the $\mathrm{B} 1 \mathrm{C}+\mathrm{B} 2 \mathrm{a}$ is not as good as that for the B1C, which we believe is due to the equal weight adopted in the data processing for the $\mathrm{B} 1 \mathrm{C}$ and the B2a, such that B1C with higher accuracy has no additional contribution.

As mentioned above, the experimental results of dual-frequency positioning are not always better than the results of single-frequency positioning due to the absence of a good stochastic model. Therefore, establishing a proper stochastic model for BDS-3 will be the focus of our next work.

\section{Acknowledgements}

The authors would like to thank the editor and reviewers for their constructive comments to improve our manuscript.

\section{Authors' contributions}

We all conceived the idea and contributed to the writing of the paper. All authors read and approved the final manuscript.

\section{Funding}

This work was partially funded by the National Natural Science Foundation of China (Grant Nos. 41604031 and 41774042), the Scientific Instrument Developing Project of the Chinese Academy of Sciences (Grant No. YJKYYQ20190063), the BDS Industrialization Project (Grant No. GFZX030302030201-2), and the National Key Research Program of China Collaborative Precision Positioning Project (Grant No. 2016YFB0501900).

\section{Availability of data and materials}

The raw data were provided by the GNSS Application and Research Group at the Institute of Geodesy and Geophysics at the Chinese Academy of Sciences in Wuhan. The raw data used in this study are available from the corresponding author upon request.

\section{Competing interests}

The authors declare that they have no competing interests.

\section{Author details}

1 State Key Laboratory of Geodesy and Earth's Dynamics, Innovation Academy for Precision Measurement Science and Technology, Chinese Academy of Sciences, Wuhan, China. ${ }^{2}$ University of Chinese Academy of Sciences, Beijing, China.

Received: 23 June 2020 Accepted: 3 November 2020 Published online: 21 December 2020

\section{References}

CSNO. (2017). BeiDou navigation satellite system signal in space interface control document open service signal B2a (Version 1.0). http://www.beidou.gov.cn/ xt/gfxz/201712/P020171226742357364174.pdf.Retrieved 27 Dec 2017.

CSNO. (2018). BeiDou navigation satellite system signal in space interface control document open service signal B3I (Version 1.0). Retrieved October 24, 2019 from https://www.beidou.gov.cn/xt/gfxz/201802/P02018020962360 1401189.pdf.

CSNO. (2019a). BeiDou navigation satellite system signal in space interface control document open service signal B1/ (Version 3.0). Retrieved October 24, 2019 from https://www.beidou.gov.cn/xt/gfxz/201902/P02019022759362 1142475.pdf.

CSNO. (2019b). BeiDou navigation satellite system signal in space interface control document open service signal B2b (Beta Version). Retrieved October 24, 2019 from https://www.beidou.gov.cn/xt/gfxz/201912/P020191227 325641753862.pdf.

Gu, S., Wang, Y., Zhao, Q., Zheng, F., \& Gong, X. (2020). BDS-3 differential code bias estimation with undifferenced uncombined model based on triplefrequency observation. Journal of Geodesy, 94, 45. https://doi.org/10.1007/ s00190-020-01364-w.

He, H., Li, J., Yang, Y., Xu, J., Guo, H., \& Wang, A. (2014). Performance assessment of single-and dual-frequency BeiDou/GPS single-epoch kinematic positioning. GPS Solutions, 18(3), 393-403. https://doi.org/10.1007/s1029 1-013-0339-3.

Hou, P., Zhang, B., Yuan, Y., Zhang, X., \& Zha, J. (2019). Stochastic modeling of BDS2/3 observations with application to RTD/RTK positioning. Measurement Science \& Technology, 30(9), 095002. https://doi.org/10.1088/13616501/ab1fad.

Jiao, G., Song, S., \& Jiao, W. (2019). Improving BDS-2 and BDS-3 joint precise point positioning with time delay bias estimation. Measurement Science \& Technology, 31(2), 025001. https://doi.org/10.1088/1361-6501/ab41cf.

Jin, S., \& Su, K. (2020). PPP models and performances from single-to quadfrequency BDS observations. Satellite Navigation, 1(1), 1-13. https://doi. org/10.1186/s43020-020-00014-y.

LV, Y., Geng, T., Zhao, Q., Xie, X., \& Zhou, R. (2020). Initial assessment of BDS-3 preliminary system signal-in-space range error. GPS Solutions, 24(1), 16. https://doi.org/10.1007/s10291-019-0928-X.

Mi, X., Zhang, B., \& Yuan, Y. (2019a). Multi-GNSS inter-system biases: Estimability analysis and impact on RTK positioning. GPS Solutions, 23(3), 81. https:// doi.org/10.1007/s10291-019-0873-8.

Mi, X., Zhang, B., \& Yuan, Y. (2019b). Stochastic modeling of between-receiver single-differenced ionospheric delays and its application to medium baseline RTK positioning. Measurement Science \& Technology, 30(9), 095008. https://doi.org/10.1088/1361-6501/ab11b5.

Mi, X., Zhang, B., Yuan, Y., \& Luo, X. (2019c). Characteristics of GPS, BDS2, BDS3 and Galileo inter-system biases and their influence on RTK positioning. Measurement Science \& Technology, 31(1), 015009. https://doi. org/10.1088/1361-6501/ab4209.

Odolinski, R., Teunissen, P. J. G., \& Odijk, D. (2014). First combined COMPASS/ BeiDou-2 and GPS positioning results in Australia. Part II: Single-and multiple-frequency single-baseline RTK positioning. Journal of Spatial Science, 59(1), 25-46. https://doi.org/10.1080/14498596.2013.866913.

Odolinski, R., Teunissen, P. J., \& Odijk, D. (2015a). Combined bds, galileo, qzss and gps single-frequency RTK. GPS Solutions, 19(1), 151-163. https://doi. org/10.1007/s10291-014-0376-6.

Odolinski, R., Teunissen, P. J. G., \& Odijk, D. (2015b). Combined GPS + BDS for short to long baseline RTK positioning. Measurement Science \& Technology, 26(4), 045801. https://doi.org/10.1088/0957-0233/26/4/045801. 
Shi, J., Ouyang, C., Huang, Y., \& Peng, W. (2020). Assessment of BDS-3 global positioning service: Ephemeris, SPP, PPP, RTK, and new signal. GPS Solution, 24, 81. https://doi.org/10.1007/s10291-020-00995-y.

Teunissen, P. J. G. (1995). The least-squares ambiguity decorrelation adjustment: A method for fast GPS integer ambiguity estimation. Journal of Geodesy, 70, 65-82. https://doi.org/10.1007/bf00863419.

Teunissen, P. J. G. (2018). Distributional theory for the DIA method. Journal of Geodesy, 92(1), 59-80. https://doi.org/10.1007/s00190-017-1045-7.

Teunissen, P. J. G., \& Verhagen, S. (2009). The GNSS ambiguity ratio-test revisited: A better way of using it. Survey Review, 41(312), 138-151. https://doi. org/10.1179/003962609X390058.

Wang, M., Wang, J., Dong, D., Meng, L., Chen, J., Wang, A., et al. (2019). Performance of BDS-3: Satellite visibility and dilution of precision. GPS Solutions, 23(2), 56. https://doi.org/10.1007/s10291-019-0847-x.

Xie, X., Geng, T., Zhao, Q., Cai, H., Zhang, F., Wang, X., et al. (2019). Precise orbit determination for BDS-3 satellites using satellite-ground and inter-satellite link observations. GPS Solutions, 23(2), 40. https://doi. org/10.1007/s10291-019-0823-5.

Yang, Y., Mao, Y., \& Sun, B. (2020). Basic performance and future developments of BeiDou global navigation satellite system. Satellite Navigation. https:// doi.org/10.1186/s43020-019-0006-0.

Yang, Y., Xu, Y., Li, J., \& Yang, C. (2018). Progress and performance evaluation of BeiDou global navigation satellite system: Data analysis based on BDS-3 demonstration system. Science China Earth Sciences, 61 (5), 614-624. https ://doi.org/10.1007/s11430-017-9186-9.

Zhang, B., Jia, X., Sun, F., Xiao, K., \& Dai, H. (2019a). Performance of BeiDou-3 satellites: Signal quality analysis and precise orbit determination. Advances in Space Research, 64(3), 687-695. https://doi.org/10.1016/j.asr.2019.05.016.

Zhang, Z., Li, B., Nie, L., Wei, C., Jia, S., \& Jiang, S. (2019b). Initial assessment of BeiDou-3 global navigation satellite system: Signal quality, RTK and PPP. GPS Solutions, 23(4), 111. https://doi.org/10.1007/s10291-019-0905-4.

\section{Submit your manuscript to a SpringerOpen ${ }^{\circ}$ journal and benefit from:}

- Convenient online submission

- Rigorous peer review

- Open access: articles freely available online

- High visibility within the field

- Retaining the copyright to your article

Submit your next manuscript at springeropen.com 